\title{
The Effects of Changes in Reaction Rates on Simulations of Nova Explosions
}

\author{
S. Starrfield*, C. Iliadis ${ }^{\dagger}$, W. R. Hix ${ }^{* *}$, F. X. Timmes ${ }^{\ddagger}$ and W. M. Sparks ${ }^{\S}$ \\ * School of Earth and Space Exploration, Arizona State University, Tempe, AZ \\ 85287-1404:sumner.starrfield@asu.edu \\ ${ }^{\dagger}$ Department of Physics and Astronomy, University of North Carolina, Chapel Hill, \\ NC27599-3255:iliadis@unc.edu \\ ** Physics Division, Oak Ridge National Laboratory, Oak Ridge, TN 37831-6354:raph@ornl.gov \\ ${ }^{\ddagger} X-2$, Los Alamos National Laboratory, Los Alamos, NM, 87545:fxt44@mac.com \\ ${ }^{\S}$ Science Applications International Corporation, San Diego CA, 92121 \& X-4, Los Alamos \\ National Laboratory, Los Alamos, NM, 87545:wms@lanl.gov
}

\begin{abstract}
Classical novae participate in the cycle of Galactic chemical evolution in which grains and metal enriched gas in their ejecta, supplementing those of supernovae, AGB stars, and WolfRayet stars, are a source of heavy elements for the ISM. Once in the diffuse gas, this material is mixed with the existing gases and then incorporated into young stars and planetary systems during star formation. Infrared observations have confirmed the presence of carbon, $\mathrm{SiC}$, hydrocarbons, and oxygen-rich silicate grains in nova ejecta, suggesting that some fraction of the pre-solar grains identified in meteoritic material come from novae. The mean mass returned by a nova outburst to the ISM probably exceeds $\sim 2 \times 10^{-4} \mathrm{M}_{\odot}$. Using the observed nova rate of $35 \pm 11$ per year in our Galaxy, it follows that novae introduce more than $\sim 7 \times 10^{-3} \mathrm{M}_{\odot} \mathrm{yr}^{-1}$ of processed matter into the ISM. Novae are expected to be the major source of ${ }^{15} \mathrm{~N}$ and ${ }^{17} \mathrm{O}$ in the Galaxy and to contribute to the abundances of other isotopes in this atomic mass range. Here, we report on how changes in the nuclear reaction rates affect the properties of the outburst and alter the predictions of the contributions of novae to Galactic chemical evolution.
\end{abstract}

Keywords: Nuclear Astrophysics; Nucleosynthesis; Nuclear Reaction Rates; Classical Novae; Cataclysmic Variables

PACS: $97.30 . \mathrm{Qt} ; 97.80 \mathrm{Gm} ; 26.30 .+\mathrm{k} ; 26.50 .+\mathrm{x}$

\section{INTRODUCTION}

The observable consequences of accretion onto white dwarfs (WDs) include the Classical $(\mathrm{CN})$, Symbiotic, and Recurrent Nova (RN) outbursts, and the possible evolution of the Super Soft, Close Binary, X-ray Sources (SSS) to Type Ia Supernova (SN Ia) explosions. This diversity of phenomena occurs because of differences in the properties of the secondary star, the mass of the WD, and the stage of evolution of the binary system (the orbital period, the luminosity of the WD and the rate of mass accretion onto the WD). A CN explosion occurs in the accreted hydrogen-rich envelope on the low-luminosity WD component of a Cataclysmic Variable (CV) system. One dimensional (1D) hydrodynamic studies, which follow the evolution of the material falling onto the WD from a bare core to the explosion, show that the envelope grows in mass until it reaches a temperature and density at its base that is sufficiently high for ignition of the hydrogen-rich fuel to occur. Both observations of the chemical abundances in $\mathrm{CN}$ ejecta and theoretical studies of the consequences of the thermonuclear runaway (TNR) in the WD envelope 
strongly imply that mixing of the accreted matter with core matter occurs at some time during the evolution to the peak of the explosion. How and when the mixing occurs is not yet known (see, e. g., Starrfield, Iliadis, and Hix 2006:S06 for a discussion).

If the bottom of the accreted layer is sufficiently degenerate and well mixed with the core, then a TNR occurs and explosively ejects core plus accreted material in a fast $\mathrm{CN}$ outburst. The evolution of nuclear burning on the WD, and the total amount of mass that it accretes and ejects depends upon: the mass and luminosity of the underlying WD, the rate of mass accretion onto the WD, the chemical composition in the reacting layers (which includes the metallicity of the CV system), the mixing history of the envelope, and the outburst history of the system.

The high levels of enrichment of novae ejecta in elements ranging from carbon to sulfur confirm that there is significant dredge-up of matter from the core of the underlying WD and enable novae to contribute to the chemical enrichment of the interstellar medium (Gehrz et al. 1998: G98). Observations of the epoch of dust formation in the expanding shells of novae allow important constraints to be placed on the dust formation process and confirm that graphite, $\mathrm{SiC}$, and $\mathrm{SiO}_{2}$ grains are formed by the outburst (G98 and references therein). It is possible that grains from novae were injected into the pre-solar nebula and can be identified with some of the pre-solar grains or "stardust" found in meteorites (Zinner 1998, Amari et al. 2001, José et al. 2004). Finally, $\gamma$-ray observations during the first several years of their outburst, done with the next generation of satellite observatories, could confirm the presence of decays from ${ }^{7} \mathrm{Be}$ and ${ }^{22} \mathrm{Na}$ (Weiss and Truran 1990; Nofar et al. 1991; Jean et al. 2000, and references therein). In the next section we report on NOVA our one-dimensional (1D) hydrodynamic computer code that we have used for the new calculations done with the reaction rate library of Iliadis (2005, private communication). We follow that with a discussion of our evolutionary results and the implications of the new rates for the nova outburst. We end with a summary.

\section{THE HYDRODYNAMIC COMPUTER CODE AND NUCLEAR REACTION RATE LIBRARIES}

NOVA is a spherically symmetric, fully implicit, Lagrangian, hydrodynamic computer code that incorporates a large nuclear reaction rate network. It is described in detail in Starrfield et al. (1998: S98), Starrfield et al. (2000:S00; and references therein). As reported in those papers, we have found that improving the opacities, equations-ofstate, and the nuclear reaction rates have had important effects on both the energetics and the nucleosynthesis. Similar results have been found in the calculations of the Barcelona group as reported elsewhere (Hernanz and Josè 2000, and references therein). Therefore, over the past few years we continued to improve the physics in NOVA and then determined the effects of the improved physics on simulations of the CN outburst (S06, and references therein). A major effort has been the effects of improving the reaction rates used in the calculations on the evolution of the $\mathrm{CN}$ outburst and the resulting nucleosynthesis. In this paper we compare our earlier studies to a recent reaction rate library of Iliadis (current as of August 2005). Since NOVA is always being updated and improved, for the work to be reported on in this paper we have made one 
major change and numerous minor changes.

The major change is that we no longer use the nuclear reaction network of Weiss and Truran (1990: WT90) but have switched to the modern nuclear reaction network of Hix and Thielemann (1999: HT99). While both networks utilize reaction rates in the common REACLIB format and perform their temporal integration using the Backward Euler method introduced by Arnett and Truran (1969: AT69), there are two important differences. First, WT90 implement a single iteration, semi-implicit backward Euler scheme, which has the advantage of a relatively small and predictable number of matrix solutions, but allows only heuristic checks that the chosen time step results in a stable or accurate solution. HT99 implement the iterative, fully implicit backward Euler scheme, repeating the Backward Euler step until convergence is achieved, providing a measure of the stability and accuracy. If convergence does not occur within a reasonable number of iterations, the time step is subdivided into smaller intervals until a converged solution is achieved. This allows the fully implicit backward Euler integration to respond to instability or inaccuracy in a way that is impossible with the semi-implicit backward Euler approach. As a result, the fully iterative approach can often safely employ larger time steps than the semi-implicit approach, obviating the speed advantage of the semiimplicit method's smaller number of matrix solutions per integration step.

Second, the HT99 network employs automated linking of reactions in the data set to the species being evolved. This is in contrast to the manual linking employed by WT90 and many older reaction networks. This automated linking helps to avoid implementation mistakes, as we discovered while performing tests of NOVA in order to understand the source of differences in the results of the simulations between two versions of the code which used the same reaction rate library but different nuclear reaction networks. In these tests, we discovered that while the REACLIB dataset used in our prior studies (S98, S00), included the pep reaction $\left(p+e^{-}+p \rightarrow d+v\right)$, it was not linked to abundance changes in the WT90 network. While for Solar modeling energy generation from the pep reaction is unimportant (but not the neutrino losses), in the WD envelope the density can reach to values of $10^{4} \mathrm{gm} \mathrm{cm}^{-3}$ which is about two orders of magnitude larger than in the core of the Sun. The increased density increases the rate of energy generation by about $40 \%$ over calculations with the pep reaction absent. The increased energy generation then has the effect of reducing the amount of accreted material since the temperature rises faster per gram of accreted material. (The effect of changes in the rate of energy generation on simulations of the $\mathrm{CN}$ outburst is discussed in detail in $\mathrm{S} 98$.) Given a smaller amount of accreted material at the time when the steep temperature rise begins in the TNR, the nuclear burning region is less degenerate and, therefore, the peak temperatures are lower for models evolved with the same nuclear reaction rate library used in our previous studies (see below).

Finally, we use the analytic fitting formulas of Itoh et al. (1996) for the neutrino energy loss rates from pair $\left(e^{+}+e^{-} \rightarrow v_{e}+\bar{v}_{e}\right)$, photo $\left(e^{ \pm}+\gamma \rightarrow e^{ \pm}+v+\bar{v}_{e}\right)$, plasma $\left(\gamma_{\text {plasmon }} \rightarrow v_{e}+\bar{v}_{e}\right)$, bremsstrahlung $\left(e^{-}+A^{Z} \rightarrow e^{-}+A^{Z}+v_{e}+\bar{v}_{e}\right)$, and recombination $\left(e_{\text {continuum }}^{-} \rightarrow e_{\text {bound }}^{-}+v_{e}+\bar{v}_{e}\right)$ processes. As stellar evolution codes generally require derivative information for the Jacobian matrix, our implementation of the Itoh et al. (1996) fitting formulas (available from http: / / www . cococubed.com) returns the neutrino loss rate and its first derivatives with respect to temperature, density, $\bar{A}$ (average 
atomic weight) and $\bar{Z}$ (average charge).

\section{EVOLUTIONARY SEQUENCES USING FOUR NUCLEAR REACTION LIBRARIES}

\subsection{The Initial Models and Libraries}

Our calculations were done with 95 zone, $1.35 \mathrm{M}_{\odot}$ complete WDs. As in S98 and S00, we assume that the material being accreted from the donor star is of Solar composition but that it has already mixed with the core material so that the actual accreting composition chosen for this study is 50\% Solar and 50\% ONeMg material. We assume a value of 2 for the mixing-length to scale height ratio $\left(l / H_{p}\right)$. All other details of our calculations (opacities, equations of state, etc.) are described in S98 and S00.

We evolved four different sequences using a different reaction rate library for each sequence but the same nuclear reaction network (HT99). The reaction rate library used in Politano et al. (1995) included the rates from Caughlan and Fowler (1988) and Thielemann et al. $(1987,1988)$. They were compiled by Thielemann and made available to Truran and Starrfield and also used for the calculations reported in WT90 (P1995 in both plots and tables). S98 used an updated reaction rate library which contained new rates calculated, measured, and compiled by Thielemann and Wiescher (labeled S98 in both plots and tables). A discussion of the improvements is provided in S98. The third library is described in Iliadis et al. (2001) and was used for the simulations in Starrfield et al. (2001). It is labeled I2001. The last library used in "This Work" is the August 2005 compilation of Iliadis. This library is an updated version of the library described in Iliadis et al. (2001) and used in Starrfield et al. (2001). A detailed description of this library will appear in Starrfield et al. (2006, in prep.). There is one additional calculation in Table 1. A comparison calculation done with the Politano et al. (1995) reaction rate library and the WT90 nuclear reaction network. As noted above, this network does not include the pep reaction and we provide it here only for comparison. We have recalculated the simulation for this paper using the same equations of state and opacities as used for the other calculations.

\subsection{The Evolutionary Results}

The initial properties of the WD are provided in the table comments. We evolved five evolutionary sequences. In all cases, we assumed an initial WD luminosity of $\sim 4 \times 10^{-3} \mathrm{~L}_{\odot}$ and a mass accretion rate of $10^{16} \mathrm{gm} \mathrm{s}^{-1}\left(1.6 \times 10^{-10} \mathrm{M}_{\odot} \mathrm{yr}^{-1}\right)$. This mass accretion rate is 5 times lower than the lowest rate used in either S98 or S00 and was chosen to maximize the amount of accreted material given the increased energy generation from the pep reaction. Numerous studies of accretion onto WDs by many different authors demonstrate that the results of the evolution depend strongly on the initial WD luminosity and mass accretion rate (c.f., Yaron et al. 2005, and references therein). 
TABLE 1. Initial Parameters and Evolutionary Results

\begin{tabular}{|c|c|c|c|c|c|}
\hline Reaction Library:* & $\mathrm{P} 1995^{\dagger}$ & $\mathrm{P} 1995^{* *}$ & S1998 & $\mathrm{I} 2001 \S$ & This Work ${ }^{\mathrm{I}}$ \\
\hline$\tau_{\mathrm{acc}}\left(10^{5} \mathrm{yr}\right)$ & 2.5 & 2.1 & 2.1 & 2.1 & 1.8 \\
\hline $\mathrm{M}_{\mathrm{acc}}\left(10^{-5} \mathrm{M}_{\odot}\right)$ & 3.9 & 3.3 & 3.3 & 3.3 & 2.8 \\
\hline $\mathrm{T}_{\text {peak }}\left(10^{6} \mathrm{~K}\right)$ & 459 & 413 & 414 & 407 & 392 \\
\hline$\varepsilon_{\text {nuc-peak }}\left(10^{17} \mathrm{erg} \mathrm{gm}^{-1} \mathrm{~s}^{-1}\right)$ & 22.8 & 8.4 & 8.6 & 4.9 & 4.4 \\
\hline $\mathrm{L}_{\text {peak }}\left(10^{5} \mathrm{~L}_{\odot}\right)$ & 8.0 & 9.6 & 8.0 & 7.3 & 5.9 \\
\hline $\mathrm{T}_{\text {eff-peak }}\left(10^{5} \mathrm{~K}\right)$ & 20 & 13 & 13 & 8.8 & 8.8 \\
\hline $\mathrm{M}_{\mathrm{ej}}\left(10^{-5} \mathrm{M}_{\odot}\right)$ & 3.3 & 2.3 & 2.3 & 2.3 & 1.7 \\
\hline $\mathrm{V}_{\max }\left(\mathrm{km} \mathrm{s}^{-1}\right)$ & 6050 & 5239 & 4755 & 4787 & 4513 \\
\hline
\end{tabular}

* The initial model for all 5 evolutionary sequences had $\mathrm{M}_{\mathrm{WD}}=1.35 \mathrm{M}_{\odot}, \mathrm{L}_{\mathrm{WD}}=4.2 \times$

$10^{-3} \mathrm{~L}_{\odot}, \mathrm{T}_{\text {eff }}=2.5 \times 10^{4} \mathrm{~K}, \mathrm{R}_{\mathrm{WD}}=2495 \mathrm{~km}$, and a central temperature of $1.2 \times 10^{7} \mathrm{~K}$

$\dagger$ Library used in Politano et al. (1995): pep reaction not included(WT90 network)

** Library used in Politano et al. (1995): pep reaction included (HT99 network)

$¥$ Library used in Starrfield et al. (1998): pep reaction included (HT99 network)

$\S$ Library described in Iliadis et al. (2001): pep reaction included (HT99 network)

II Iliadis (2005: this work) library: pep reaction included (HT99 network)

We use the same composition for the accreting material as in Politano et al. (1995; see also: S98; S00; and Starrfield et al. 2001: a mixture of half-solar and half-ONeMg by mass fraction). By using this composition, we assume that core material has mixed with accreted material from the beginning of the evolution. This composition also effects the amount of accreted mass at the peak of the TNR since it has a higher opacity than if no mixing were assumed. The results of our evolutionary calculations are given in Table 1 and 2. Table 1 gives the initial parameters and evolutionary results and Table 2 gives the abundances of the ejected material (by mass) for the 4 different simulations. We do not report the abundance results for the sequence done without the pep reaction since this calculation is not realistic. The evolutionary parameters are provided only to demonstrate the effects of including this reaction on the evolution.

The rows in Table 1 are the reaction rate library, the accretion time to the $\operatorname{TNR}\left(\tau_{\mathrm{acc}}\right)$, the accreted mass $\left(\mathrm{M}_{\mathrm{acc}}\right)$, peak temperature in the TNR $\left(\mathrm{T}_{\text {peak }}\right)$, peak rate of energy generation during the TNR, $\left(\varepsilon_{\text {nuc-peak }}\right)$, peak luminosity $\left(\mathrm{L}_{\text {peak }}\right)$, peak effective temperature $\left(\mathrm{T}_{\text {eff-peak }}\right)$, ejected mass $\left(\mathrm{M}_{\mathrm{ej}}\right)$, and the peak expansion velocity after the radii of the surface layers have reached $\sim 10^{13} \mathrm{~cm}\left(\mathrm{~V}_{\max }\right)$. By this time the outer layers are optically thin, have far exceeded the escape velocity, and there is no doubt that they are escaping.

As noted above, we provide two different columns for Politano et al. (1995). The first, with the superscript $\dagger$, is taken from a calculation done for this paper using the Politano et al. library and the WT90 network in NOVA. The second, with the superscript **, uses the same reaction rate library as Politano et al. but the energy generation and nucleosynthesis is obtained with the HT99 network. These two columns, therefore, show the effects of including the pep reaction on the TNR simulations.

Table 1 shows that the largest change in the results of the evolution occurs with the inclusion of the pep reaction. If we compare $\mathrm{P} 1995^{\dagger}$ and $\mathrm{P} 1995^{* *}$, then the $\sim 40 \%$ increase in energy production from just adding the pep reaction to the network results in a decrease of $\sim 19 \%$ in both accretion time and accreted mass. The large change must 


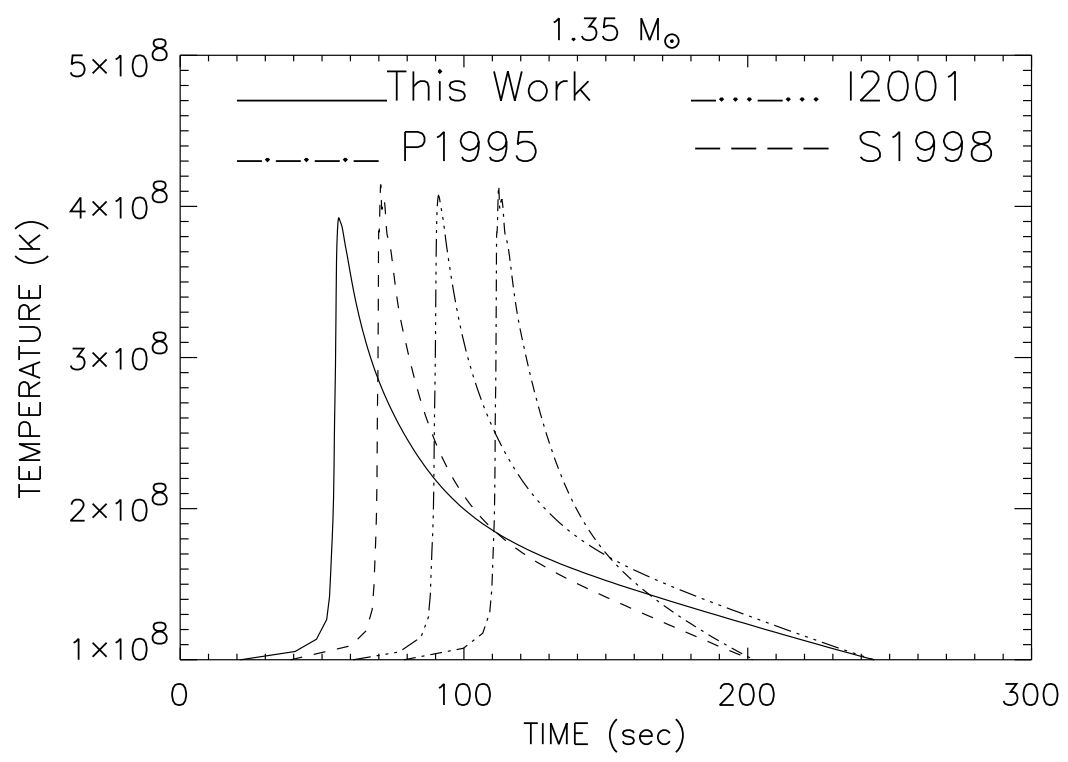

FIGURE 1. The variation with time of the temperature in the deepest hydrogen-rich zone around the time when peak temperature occurs. We have plotted the results for four different simulations on a $1.35 \mathrm{M}_{\odot}$ WD. The identification with calculations done with a specific library is given on the plot. In this plot and all following plots, S1998 refers to Starrfield et al. (1998:S98), P1995 refers to Politano et al. (1995), I2001 refers to Iliadis et al. (2001), and This Work refers to this paper. The details of the associated reaction rate library are given in the text. The curve for each sequence has been shifted slightly in time to improve its visibility.

be caused by the addition of the pep reaction since the two reaction rate libraries are otherwise identical. Because there is less accreted mass on the WD at the time of the TNR (comparing the sequences done with the pep reaction included to the one without it), the peak temperatures do not reach to as high values as in the sequence with the pep reaction absent. If we compare the accretion time and accreted mass for the four sequences with the pep reaction included, we see that changes in the nuclear reaction rate library have a sizable effect. In addition, there have been some small changes to the reaction rates in the proton-proton chain and that is where the sequences spend most of their time during the accretion phase.

Figure 1 shows the variation of temperature with time for the deepest hydrogen-rich zone and we plot only the simulations done with the pep reaction included. The specific evolutionary sequence is identified on the plot. The reference to the reaction network used for that calculation is given in the caption for Figure 1. The time coordinate is arbitrary and chosen to clearly show each curve. This figure shows that there are important differences between the four simulations. Peak temperature drops from about 413 million degrees to 392 million degrees and peak nuclear energy generation drops by about a factor of 2.5 from the oldest library to the newest library $\left(8.4 \times 10^{17} \mathrm{erg} \mathrm{gm}^{-1} \mathrm{~s}^{-1}\right.$

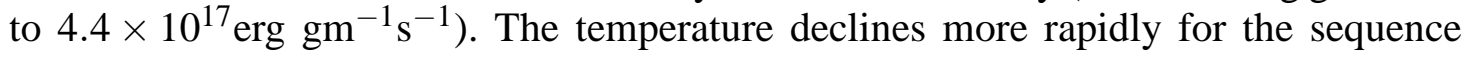
computed with the oldest reaction library (Politano et al. 1995) because it exhibited a larger release of nuclear energy throughout the evolution which caused the overlying zones to expand more rapidly and the nuclear burning layers to cool more rapidly. In 
TABLE 2. Comparison of the Ejecta Abundances for $1.35 \mathrm{M}_{\odot}$ White Dwarfs (All abundances are mass fraction)

\begin{tabular}{lcccc}
\hline Reaction Library: & P1995* & S1998 & I2001 & This Work \\
\hline $\mathrm{H}$ & 0.27 & 0.27 & 0.27 & 0.28 \\
${ }^{4} \mathrm{He}$ & 0.18 & 0.18 & 0.17 & 0.17 \\
${ }^{12} \mathrm{C}$ & $8.0 \times 10^{-3}$ & $1.2 \times 10^{-2}$ & $8.0 \times 10^{-3}$ & $6.2 \times 10^{-3}$ \\
${ }^{13} \mathrm{C}$ & $2.8 \times 10^{-3}$ & $4.0 \times 10^{-3}$ & $2.4 \times 10^{-3}$ & $2.4 \times 10^{-3}$ \\
${ }^{14} \mathrm{~N}$ & $4.3 \times 10^{-3}$ & $4.8 \times 10^{-3}$ & $4.3 \times 10^{-3}$ & $8.4 \times 10^{-3}$ \\
${ }^{15} \mathrm{~N}$ & 0.11 & 0.11 & $6.8 \times 10^{-2}$ & $6.0 \times 10^{-2}$ \\
${ }^{16} \mathrm{O}$ & $1.2 \times 10^{-3}$ & $1.1 \times 10^{-3}$ & $2.4 \times 10^{-3}$ & $2.4 \times 10^{-3}$ \\
${ }^{17} \mathrm{O}$ & $1.1 \times 10^{-3}$ & $1.0 \times 10^{-3}$ & $5.9 \times 10^{-2}$ & $6.7 \times 10^{-2}$ \\
${ }^{18} \mathrm{O}$ & $7.8 \times 10^{-3}$ & $6.7 \times 10^{-3}$ & $3.0 \times 10^{-3}$ & $1.5 \times 10^{-3}$ \\
${ }^{18} \mathrm{~F}$ & $2.5 \times 10^{-3}$ & $2.3 \times 10^{-3}$ & $9.3 \times 10^{-4}$ & $5.9 \times 10^{-4}$ \\
${ }^{22} \mathrm{Na}$ & $3.5 \times 10^{-2}$ & $5.1 \times 10^{-2}$ & $3.0 \times 10^{-2}$ & $2.3 \times 10^{-2}$ \\
${ }^{24} \mathrm{Mg}$ & $2.8 \times 10^{-3}$ & $1.9 \times 10^{-3}$ & $2.1 \times 10^{-3}$ & $1.9 \times 10^{-3}$ \\
${ }^{26} \mathrm{Mg}$ & $1.6 \times 10^{-2}$ & $1.2 \times 10^{-2}$ & $2.2 \times 10^{-3}$ & $1.5 \times 10^{-3}$ \\
${ }^{26} \mathrm{Al}$ & $2.8 \times 10^{-3}$ & $2.1 \times 10^{-3}$ & $2.7 \times 10^{-3}$ & $3.0 \times 10^{-3}$ \\
${ }^{27} \mathrm{Al}$ & $2.8 \times 10^{-2}$ & $3.4 \times 10^{-2}$ & $1.4 \times 10^{-2}$ & $1.4 \times 10^{-2}$ \\
${ }^{28} \mathrm{Si}$ & $2.3 \times 10^{-2}$ & $3.5 \times 10^{-2}$ & $2.7 \times 10^{-2}$ & $2.9 \times 10^{-2}$ \\
${ }^{29} \mathrm{Si}$ & $6.3 \times 10^{-3}$ & $7.0 \times 10^{-3}$ & $1.9 \times 10^{-2}$ & $1.8 \times 10^{-2}$ \\
${ }^{30} \mathrm{Si}$ & $2.3 \times 10^{-2}$ & $2.4 \times 10^{-2}$ & $3.1 \times 10^{-2}$ & $3.8 \times 10^{-2}$ \\
${ }^{31} \mathrm{P}$ & $3.0 \times 10^{-2}$ & $3.0 \times 10^{-2}$ & $4.3 \times 10^{-2}$ & $3.7 \times 10^{-2}$ \\
${ }^{32} \mathrm{~S}$ & $2.1 \times 10^{-2}$ & $2.8 \times 10^{-2}$ & $3.9 \times 10^{-2}$ & $4.0 \times 10^{-2}$ \\
${ }^{34} \mathrm{~S}$ & $1.5 \times 10^{-3}$ & $1.3 \times 10^{-3}$ & $1.3 \times 10^{-3}$ & $7.2 \times 10^{-4}$ \\
${ }^{36} \mathrm{Ar}$ & $6.1 \times 10^{-4}$ & $2.2 \times 10^{-4}$ & $1.5 \times 10^{-4}$ & $6.8 \times 10^{-5}$ \\
${ }^{40} \mathrm{Ca}$ & $2.1 \times 10^{-5}$ & $2.4 \times 10^{-5}$ & $2.4 \times 10^{-5}$ & $1.8 \times 10^{-5}$ \\
\hline
\end{tabular}

* Library used in Politano et al. (1995): pep reaction included (HT99 network)

$\dagger$ Library used in Starrfield et al. (1998): pep reaction included (HT99 network)

** Library described in Iliadis et al. (2001): pep reaction included (HT99 network)

$\ddagger$ Iliadis (2005: this work) library: pep reaction included (HT99 network)

contrast, the newest library, with the smallest expansion velocities, cools slowly.

The differences in total nuclear energy generation $\left(\mathrm{L} / \mathrm{L}_{\odot}\right)$ as a function of time for each mass is shown in Figure 2. The time coordinate is consistent with that used in Figure 1. Note that peak nuclear energy production for the latest library is definitely lower than seen in the earlier libraries. The changes in the libraries are more important for the more massive isotopes and become more important as higher temperatures are reached.

The abundance predictions, for the ejected material, from our four evolutionary sequences are given as mass fraction in Table 2 . Here we discuss only the most important nuclei. The increase in the ${ }^{4} \mathrm{He}$ abundance is small compared to the observed helium abundances in $\mathrm{CN}$ ejecta which can reach, if not exceed, 0.5 (G98). The large helium abundance, in combination with the large observed CNO abundances, is strong evidence for mixing of the accreted material with layers in the WD underlying the accreting material at some time during the outburst. The large helium abundances observed in RN such as U Sco or V394 CrA suggest that mixing has also occurred in these systems even if their total $\mathrm{CNO}$ abundances are not dramatically enriched over solar. Examining the 


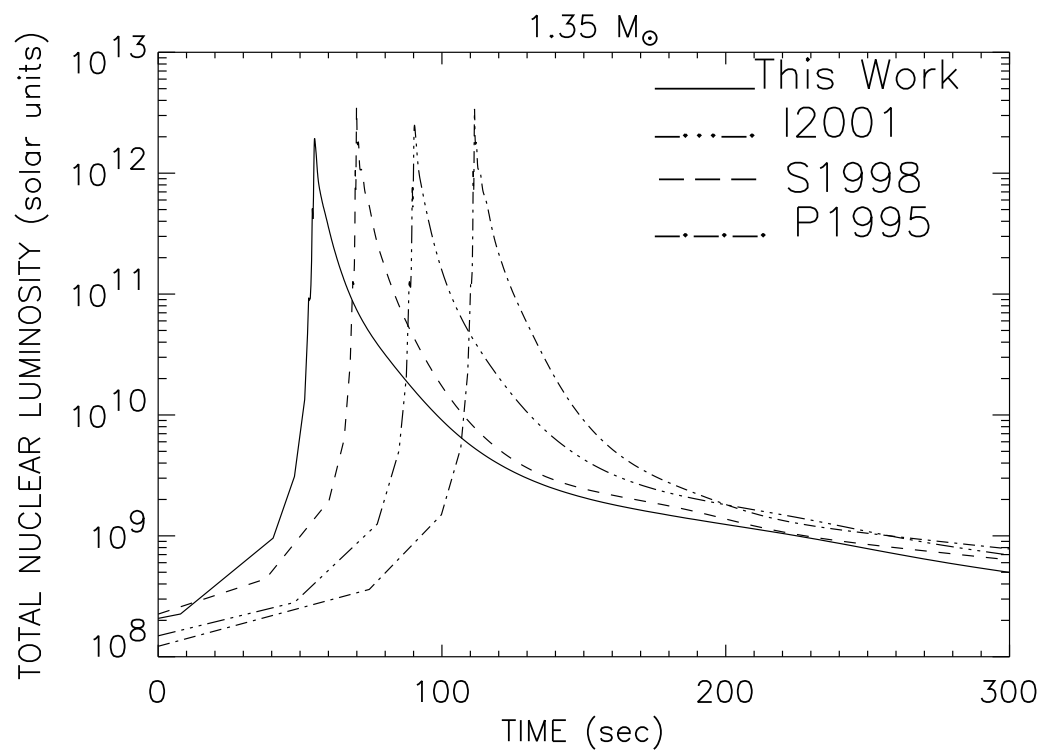

FIGURE 2. The variation with time of the total nuclear luminosity $\left(\mathrm{erg} \mathrm{s}^{-1}\right)$ in solar units $\left(\mathrm{L}_{\odot}\right)$ around the time of peak temperature during the TNR on a $1.35 \mathrm{M}_{\odot} \mathrm{WD}$. We integrated over all zones taking part in the explosion. The identification with each library is given on the plot. The time coordinate is chosen to improve visibility.

behavior of the individual abundances, we see that ${ }^{12} \mathrm{C}$ and ${ }^{13} \mathrm{C}$ are virtually unchanged by the updated reaction rates. In contrast, the abundance of ${ }^{14} \mathrm{~N}$ nearly doubles and that of ${ }^{15} \mathrm{~N}$ decreases by a factor of two going from the first to the latest reaction rate library. ${ }^{16} \mathrm{O}$ also doubles in abundance while ${ }^{17} \mathrm{O}$ grows by a factor of 60 and becomes the most abundant of the CNO nuclei in the ejecta. For this WD mass, the C/O ratio is 0.12 .

The abundance of ${ }^{22} \mathrm{Na}$ decreases with the library update and ${ }^{24} \mathrm{Mg}$ is severely depleted by the TNR. ${ }^{26} \mathrm{Al}$ is unchanged by the changes in the reaction rates while the abundance of ${ }^{27} \mathrm{Al}$ drops by a factor of two. This result implies that TNRs on more massive WDs eject about the same fraction of ${ }^{26} \mathrm{Al}$ as ${ }^{27} \mathrm{Al}$. We also find that contrary to Politano et al. (1995) that the amount of ${ }^{26} \mathrm{Al}$ ejected is virtually independent of WD mass. All the Si isotopes $\left({ }^{28} \mathrm{Si},{ }^{29} \mathrm{Si}\right.$, and $\left.{ }^{30} \mathrm{Si}\right)$ are enriched in the calculations done with the latest library. Finally, while the ejecta abundances of ${ }^{36} \mathrm{Ar}$, and ${ }^{40} \mathrm{Ca}$ have declined as the reaction rate library has been improved, they are all produced in the nova TNR since their final abundances exceed the initial abundances.

\section{SUMMARY}

In this paper we examined the consequences of improving the nuclear reaction library on our simulations of TNRs on $1.35 \mathrm{M}_{\odot}$ WDs. We have found that the changes in the rates have affected the nucleosynthesis predictions of our calculations but not, to any great extent, the gross features of the evolution. In addition, we have used a lower mass accretion rate than in our previous studies in order to accrete (and eject) more material. This has, as expected, caused the peak values of some important parameters 
to increase over our previous studies at the same WD mass. However, because some important reaction rates have declined in the new reaction rate library this has not increased the abundances for nuclei above aluminum and, in fact, they have declined

while the abundances of both ${ }^{26} \mathrm{Al}$ and ${ }^{27} \mathrm{Al}$ have increased. In contrast, the abundance of ${ }^{22} \mathrm{Na}$ has declined from the values predicted in our earlier work.

\section{ACKNOWLEDGMENTS}

S. Starrfield acknowledges partial support from NSF and NASA grants to ASU. He also thanks J. Aufdenberg and ORNL for generous allotments of computer time. WRH is partly supported by the NSF under contracts PHY-0244783 and and by the DOE, through the Scientific Discovery through Advanced Computing Program. ORNL is managed by UT-Battelle, LLC, for the U.S. DOE under contract DE-AC05-00OR22725.

\section{REFERENCES}

1. Amari, S., Gao, X. Nittler, L. R., Zinner, E., José, J. Hernanz, M. Lewis, R. 2001, ApJ, 551, 1065

2. Arnett, W. \& Truran, J. 1969, ApJ, 157, 339

3. Caughlan, G. R., \& Fowler, W.A., 1988, At. Data Nucl. Data Tables, 40, 283

4. Gehrz, R.D., Truran, J.W., Williams, R.E., \& Starrfield, S. 1998, PASP, 110, 3

5. Hix, W. R. \& Thielemann, F.-K. 1999, ApJ, 511, 862

6. Hernanz, M., \& José, J. 2000, in Cosmic Explosions, Ed. S. S. Holt \& W. W. Zhang, AIP Conference Proceedings \# 522, p. 339

7. Iliadis, C., D’Auria, J.M., Starrfield, S., Thompson, W.J., \& Wiescher, M. 2001, ApJS, 134, 151

8. Itoh, N. et al. 1996, ApJS, 102, 411

9. Jean, P., Hernanz, M., Gómez-Gomar, J., \& José, J. 2000, MNRAS, 319, 350

10. José, J. Hernanz, M., Amari,S., Lodders, K., Zinner, E. 2004, ApJ, 612, 614

11. Nofar, I., Shaviv, G., \& Starrfield, S. 1991, ApJ, 369, 440

12. Politano, M., Starrfield, S., Truran, J. W., Weiss, A., \& Sparks, W. M. 1995, ApJ, 448, 807

13. Starrfield, S., Iliadis, C., \& Hix, W. R. 2006, in Classical Nova II, ed. M. F. Bode \& A. Evans, Cambridge, University Press, in press.

14. Starrfield, S., Iliadis, C., Truran, J. W., Wiescher, M., \& Sparks, W. M. in Proceedings of the 6th International Conference on Nuclei in the Cosmos 2001, ed. J. Christensen-Dalsgaard \& K. H. Langanke, (North-Holland, Elsevier, New York,) Nuclear Physics A, 688, 110c

15. Starrfield, S., Sparks, W. M., Truran, J.W., Wiescher, M.C. 2000, ApJS, 127, 485

16. Starrfield, S., Truran, J.W., Wiescher, M.C., \& Sparks, W. M. 1998, MNRAS, 296, 502

17. Thielemann, F.-K., Arnould, M., \& Truran, J. W. 1987, in advances in Nuclear Astrophysics, ed. E. Vangioni-Flam, Gif-sur-Yvette, Editions Frontieres, 525

18. Thielemann, F.-K., Arnould, M., \& Truran, J. W. 1988, in Capture Gamma-Ray Spectroscopy, ed. K. Abrahams \& P. Van assche, Bristol, IOP, 730

19. Weiss, A., \& Truran, J. W. 1990, A\&A, 238, 178

20. Yaron, O., Prialnik, D., Shara, M. M., Kovetz, A. 2005, ApJ, 623, 398

21. Zinner, E. 1998, Annual Review of Earth and Planetary Sciences, 26, 147 